\title{
The T.O.S.CA. Project: Research, Education and Care
}

\section{Progetto T.O.S.CA.: Ricerca, Formazione e Cura}

\author{
Eduardo Bossone1, Giuseppe Limongelli2, Graziella Malizia3, Francesco Ferrara4, \\ Olga Vriz6, Rodolfo Citro1, Alberto Maria Marra4, Michele Arcopinto4, Emanuele Bobbio4, \\ Domenico Sirico4, Luigi Caliendo5, Andrea Ballotta7, Antonello D’Andrea2, \\ Alessandro Frigiola7, Jorgen Isgaard8, Luigi Saccà4 and Antonio Cittadini4. \\ On behalf of T.O.S.CA. investigators
}

\begin{abstract}
The T.O.S.CA. Project: Research, Education and Care. E. Bossone, G. Limongelli, G. Malizia, F. Ferrara, O. Vriz, R. Citro, A.M. Marra, M. Arcopinto, E. Bobbio, D. Sirico, L. Caliendo, A. Ballotta, A. D'Andrea, A. Frigiola, J. Isgaard, L. Saccà and A. Cittadini. On behalf of T.O.S.CA. investigators.

Despite recent and exponential improvements in diagnostic-therapeutic pathways, an existing "GAP" has been revealed between the "real world care" and the "optimal care" of patients with chronic heart failure (CHF). We present the
\end{abstract}

T.O.S.CA. Project (Trattamento Ormonale dello Scompenso CArdiaco), an Italian multicenter initiative involving different health care professionals and services aiming to explore the CHF "metabolic pathophysiological model" and to improve the quality of care of $\mathrm{HF}$ patients through research and continuing medical education.

Keywords: heart failure, metabolic pathophysiological model, quality of care, TOSCA.

Monaldi Arch Chest Dis 2011; 76: 198-203.

\footnotetext{
1 Department of Cardiology and Cardiac Surgery, University Hospital "Scuola Medica Salernitana", Salerno, Italy.

2 Department of Cardiothoracic Sciences, Monaldi Hospital, Second University of Naples, Italy.

3 Division of Endocrinology, Ospedali Riuniti, Villa Sofia-Cervello, Palermo, Italy.

4 Department of Internal Medicine, Cardiovascular and Immunological Sciences, University Federico II, Naples, Italy.

3 Division of Cardiology, San Leonardo Hospital, Castellammare di Stabia, Italy.

6 Department of Emergency and Cardiology, San Antonio Hospital, San Daniele del Friuli, Udine, Italy.

7 Department of Cardiac Surgery I.R.C.C.S. Policlinico San Donato, Milan, Italy.

8 Department of Internal Medicine, Sahlgrenska Academy Göteborg University, Sweden.
}

Corresponding author: Prof. Antonio Cittadini; Department of Internal Medicine and Cardiovascular Sciences, University Federico II, Via Pansini, 5; I-80131 Naples, Italy; Tel/Fax: +39-081-7464365; E-mail address: antonio.cittadini@unina.it

\section{Introduction}

Chronic Heart Failure (CHF) is a growing public health problem impacting heavily on patient survival, quality of life and healthcare costs. Due mainly to coronary artery disease [1], hypertension, valve disease and cardiomyopathies, it is characterized by (a) shortness of breath at rest or during exertion, and fatigue; (b) signs of fluid retention such as pulmonary congestion or ankle swelling; (c) and an objective evidence of an abnormality of the structure or function of the heart at rest [2].

The incidence and prevalence $(2-3 \% ; 10-20 \%$ after 75 years of age) are clearly increasing in industrialized countries [2,3]. According to the European Society of Cardiology roughly 15 million patients were diagnosed with CHF out of a population of 900 million people.

However, despite considerable investment in research and guidelines dissemination by several scientific societies, the treatment gap of CHF continues to widen between evidence-based approach and practice reality across the continuum of care, resulting in an unsustainable high morbidity and mortality along with an increasing economic and social burden [4-8].

We herein present the design and the aims of The T.O.S.CA. Project (Trattamento Ormonale dello Scompenso CArdiaco): an Italian multicenter initiative aiming to explore the CHF "metabolic pathophysiological model" and to improve the quality of care through the implementation of a dedicated continuing medical educational program.

\section{Research}

Despite different hypotheses, no single pathophysiologic paradigm of CHF has been definitively established. One logical explanation of the inability to define the syndrome of CHF in precise mechanistic models is that the clinical syndrome of $\mathrm{CHF}$ almost certainly represents the summation of multiple anatomic, functional, and biological alterations that interact together in an complex way. Thus, it is not surprising that investigators employing simple models in an attempt to describe the syndrome of HF have failed [9]. 
Nowadays, the most accepted hypothesis explaining CHF pathophysiology and its progression is the "neurohormonal model". According to this paradigm, heart failure progresses as a result of the overexpression of biologically active molecules that exert toxic effects on the heart and circulation. A variety of molecules including norepinephrine, angiotensin II, endothelin, aldosterone, and tumor necrosis factor have been implicated as some of the factors that contribute to disease progression in the failing heart [9].

The evidence in support of this point of view is derived from many experimental and clinical studies showing that overexpression of active molecules reported above can contribute to disease progression independently of the hemodynamic status of the patient and that antagonizing neurohormones improve clinical outcomes of patients with CHF. Indeed, the "neurohormonal axis" is the main target of medical treatments proved to be efficacious in CHF, such as ACEI, beta blockers and spironolacton/eplerenon [10].

However, the "neurohormonal model" displayed several pitfalls. A complete inhibition of hormonal axes involved in CHF progression is difficult to obtain for dose-limiting effects of drugs and also for alternative metabolic pathways that are not antagonized by conventional therapy. On the other hand, other unknown molecular interactions potentially involved in the progression of CHF are likely to be involved.

Despite the effectiveness of the neurohormonal model to explain disease progression and the many insights that it provided for the development of new therapies, there is an increasing clinical evidence that suggests that our current models fail to completely explain disease progression.

The observation that the survival curves become parallel following neurohormonal antagonism suggests that there may be an attenuation or loss of effectiveness of neurohormonal antagonism as heart failure progresses. Thus, neurohormonal models may be necessary but not sufficient to explain all the aspects of disease progression of the failing heart.

Based on these observations and on the poor prognosis despite optimal therapies, alternative mechanisms that contribute to CHF progression are being actively explored. In this regard, recent evidence suggests that a metabolic imbalance occurs in $\mathrm{CHF}$, characterized by a predominance of the catabolic status over anabolic drive. The most impressive example is seen in end-stage CHF known as "cardiac cachexia" characterized by sustained weight loss, particularly lean mass, and rapid deterioration of clinical conditions, attributed to a prevalence of catabolic pathways. Whether such hormonal imbalance is a mere epiphenomenon or an important pathophysiological mechanism in CHF progression is still matter of debate. The main evidence concerning anabolic deficiencies in CHF patients is described in a recent study by Jankowska et al. [11]; specifically, deficit of each anabolic axis (adrenal, gonadal and somatotropic axes) represents an independent marker of poor outcome in CHF patients and the coexistence of more than one deficiency identifies a subgroup of patients with a higher mortality.
The most involved hormonal axes include growth hormone $(\mathrm{GH})$, its tissue effector insulinlike growth factor-1 (IGF-1), thyroid hormone, insulin and anabolic steroids. Taken together, these alterations could be recognised as a multiple hormonal and metabolic deficiency syndrome (MHD) in HF patients. MHD bears a significant impact on cardiac performance and HF progression [12].

These considerations provide novel and interesting opportunities including: (a) The identification of new prognostic markers; (b) The implementation of knowledge about multiple hormonal deficiency syndrome (MHD); (c) The basis for new therapeutic approaches in this regards our group has some recent experiences in $\mathrm{GH}$ replacement therapy.

Chief among the hormonal systems the GH/IGF1 axis play a pivotal role, being the most powerful anabolic pathway in the human body. In fact, GH and IGF-1 are essential for preserving both cardiac morphology and performance in adult life. The finding of low IGF-1 levels in CHF patients is relevant for many reasons. First, individuals with low IGF-1 levels undergo cardiovascular alterations that are corrected by replacement therapy; in this regards our group has some recent experiences in GH replacement therapy [13]. Second, large population-based studies show that a low level of IGF-1 is predictive of HF. Finally, low IGF-1 levels are associated with reduced skeletal muscle performance and endothelial dysfunction [14].

Thus, study of GH/IGF-1 axis represents the main topic of T.O.S.CA. investigations.

Preliminary insights from T.O.S.CA. project about clinical status and cardiovascular performance in patients with low IGF-1 levels were presented at the recent European Congress of Cardiology [15].

\section{Aims of T.O.S.CA. Project - Research:}

The T.O.S.CA. project therefore represents a unique opportunity to create a large database from several centers both in Italy and abroad in order to respond to some fundamental pathophysiological questions. First, what are the reference values of the main anabolic axes in a large Italian CHF population, coming from different regions of our country? What is the actual prevalence of multiple hormone deficiencies in CHF? Second, we will evaluate whether anabolic deficiencies identify subgroups of CHF patients with worse clinical status and more impaired LV architecture, function and cardiopulmonary performance. Third and more importantly, we will be able to address the question of whether single or multiple hormone deficiencies affect heart failure progression and poor outcome.

Smaller case-control studies will address more specific topics such as the relation between direct assessment of myocardial ultrastructure and gene expression profile, and the anabolic/catabolic balance, evaluating myocardial biopsies of CHF patients undergoing cardiac surgery.

\section{Education}

Despite the recent and exponential improvements in diagnostic-therapeutic pathways, an existing "GAP" has been revealed from the "real world 
care" and the "optimal care" of patients with $\mathrm{CHF}$ [16-28]. A recent Italian multicentric populationbased study has confirmed the "GAP" in health status among socioeconomic groups particularly evident for CHF characterized by an excess of unnecessary hospitalization [29].

In Italy the educational health program (defined as E.C.M: Continuing Medical Education) consists of theoretical and practical activities promoted by agencies and scientific societies and regulated by specific legislation (DLgs 229/99 and L. 238/00). In particular, the purpose of the T.O.SCA project educational branch is to improve the quality of care of $\mathrm{CHF}$ patients (timeliness, efficiency and effectiveness) in different clinical settings. It consists of a specialized E.C.M.-meta-model designed by a dedicated multidisciplinary team and applied through an intensive three phases educational year (Fig. 1).

Phase 1: Pre-T.O.SCA: (a) Selection of participant hospitals and investigators; (b) Investigators' training (meeting-tutorial-web site); (c) Presentation and beginning of the project; (d) Analysis "status quo" of actual diagnostic-therapeutic pathways. Data (i.e. medical history, symptoms and signs, electrocardiographic, chest X Ray, echo-Doppler and other imaging results, in -hospital treatment disposition, follow-up) will to be collected on a standard case-report form and then forwarded to the T.O.S.CA. registry coordinating center for analysis. Quality of care is going to be assessed by measuring the use of "key indicators" as for current ESC guidelines.

Phase 2: T.O.SCA Implementation: (e) Improvement of adhesion to the guidelines through dedicated tools;(f) Weekly-monthly meeting (ECM) and quality control.

Phase 3: post-T.O.SCA: (g) Analysis post guidelines implementation. (h) Presentation and discussion of results.

\section{Care}

The actual picture of CHF in Europe and Italy is predominantly represented by continuous acute hospital admissions, poor prognosis, an ongoing drain of health care expenditure and discomfort for the patients and care givers [30-33]. Thus, in order to optimize the medical management, reduce the hospitalization and readmission rate, improve and maintain optimum quality of life, and balance the cost/benefits ratio, the European Society of Cardiology guidelines recommend the implementation of structured multisciplinary CHF disease-management program (Class of recommendation I, level of evidence A) taking into account patient needs, financial resources, available personnel, and administrative policies [2]. In this regard the T.O.S.CA. team has developed a meta-model of structured CHFMG (Chronic Heart Failure Management Program) (Fig. 2) on the area of Amalfi Coast in Southern Italy as a continuum between the community hospitals and the territorial health system serving 110.000 habitants among 13 cities. The CHF out-patient clinic represents the platform of the Amalfi Coast CHFMG, directed by clinical cardiologist and coordinated by registered nurse, along with multidisciplinary team. Patient-first contact with CHFMG may be during hospitalization or as specific request from primary care physician. During the first outpatient visit the cardiologist will assess the clinical status and current therapy, design an effective follow-up strategy such as ambulatory care, day service, day hospital [35-37]. A tertiary level hospital constitutes the referral center for invasive and surgical treatments (revascularization procedures, valvular and ventricular surgery, cardiac resynchronization therapy, implantable cardioverter defibrillator, heart transplantation and ventricular assist devices). Cardiac rehabilitation and genetic counseling are also provided when indicated. 


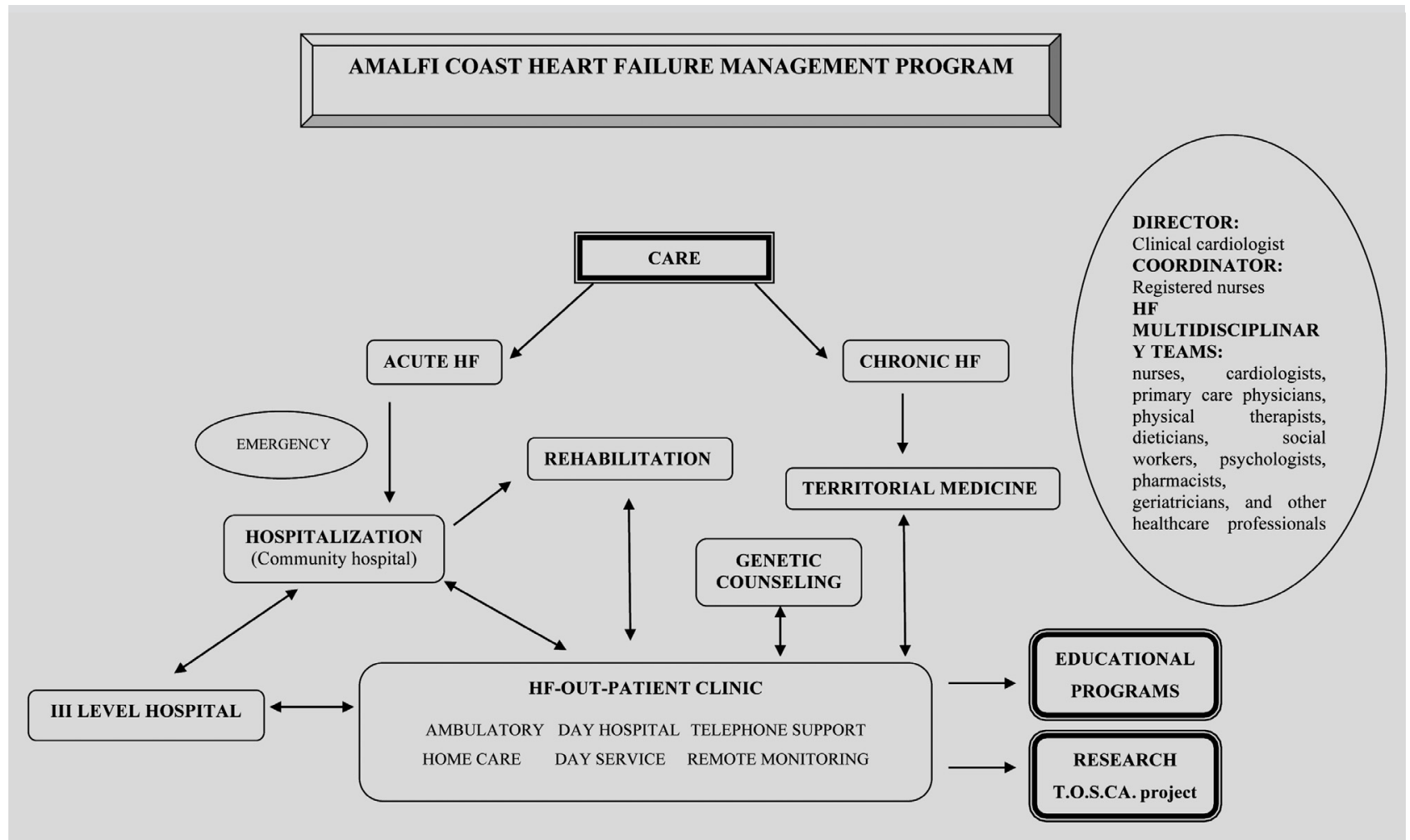

Figure 2.

\section{Genetic Counselling and "Phenotyping"}

In this regard, genetic counseling represents an important preliminary part of the study cohort selection process. Indeed, as mentioned above, the clinical syndrome of CHF represent the "summa" of multiple anatomic, functional, and molecular alterations that contribute to initiate (preclinical phase), manifest (onset), and worsen (progression to end stage) HF. On this ground, the clinical attempt to define "subgroup of CHF syndromes" helps to better characterize the clinical phenotype. In clinical practice, this will be realized by:

(a) the study of the family tree (sudden deaths, transplants, cardiomyopathies/myopathies); (b) clinical exclusion of metabolic-mitochondrial (systemic), and neuromuscular involvement ("clinical red flags"); (c) integrated imaging (new echo technologies and MRI); (d) phenotype-guided molecular analysis (in selected cases).

Finally, the phenotype characterization will recognize these groups:

(1) ischemic cardiomyopathy, (2) familial dilated cardiomyopathy, (3) non familial, dilated cardiomyopathy (idiopathic), (4) myocarditis and autoimmune cardiomyopathy

Exclusion criteria will be: diagnosis of neuromuscular (ie: dystrophynopathy, laminopathy, etc), mitochondrial (i.e.: MELAS and MERRF syndrome), and toxic (i.e. cocaine or pheocromocytoma) cardiomyopathy [38-39].

\section{Conclusions}

In conclusion, the T.O.S.CA. project may represent a challenging "learning by doing" experience involving different health care professionals and ser- vices and aiming to explore the CHF "metabolic pathophysiological model" and to improve the quality of care of CHF patients through research and continuing medical education.

\section{Riassunto}

Nonostante $i$ recenti ed esponenziali progressi ottenuti nel campo diagnostico-terapeutico, è stato rilevato un "GAP" esistente tra la pratica clinica e la gestione ideale dei pazienti con Insufficienza Cardiaca Cronica (ICC). In questo lavoro viene presentato il progetto T.O.S.CA. (Trattamento Ormonale dello Scompenso CArdiaco), un'iniziativa italiana multicentrica che coinvolge differenti professionisti e servizi sanitari finalizzata all' esplorare il cosiddetto "modello fisiopatologico e metabolico" e nel migliorare la qualità del trattamento dei pazienti affetti da ICC attraverso protocolli di ricerca ed aggiornamento medico continuo.

\section{References}

1. Fox KF, Cowie MR, Wood DA, Coats AJ, Gibbs JS, Underwood SR. Coronary artery disease as the cause of incident heart failure in the population. Eur Heart $J$ 2001; 22: 228-236.

2. Dickstein K, Cohen-Solal A, Filippatos G, et al. ESC Guidelines for the diagnosis and treatment of acute and chronic heart failure 2008. Eur Heart J 2008; 29: 2338-2442.

3. Senni M, Tribouilloy CM, Rodeheffer RJ, et al. Congestive heart failure in the community: trends in incidence and survival in a 10-year period. Arch Intern Med 1999; 159: 29-34.

4. Stewart S, Jenkins A, Buchan S, McGuire A, Capewell S, McMurray JJ. The current cost of heart failure to the National Health Service in the UK. Eur J Heart Fail 2002; 4: 361-371. 
5. McKee PA, Castelli WP, McNamara PM, Kannel WB (1971) The natural history of congestive heart failure: the Framingham study. N Engl J Med 285: 1441-1446.

6. Stewart S, MacIntyre K, Hole DJ, Capewell S, McMurray JJ. More 'malignant' than cancer? Five-year survival following a first admission for heart failure. Eur J Heart Fail 2001; 3: 315-322

7. Cowie MR, Wood DA, Coats AJ, et al. Survival of patients with a new diagnosis of heart failure: a population based study. Heart 2000; 83: 505-510.

8. Cleland JG, Gemmell I, Khand A, et al. Is the prognosis of heart failure improving? Eur Heart Fail 1999; 1: 229-241.

9. Mann DL. Mechanisms and models in heart failure: A combinatorial approach. Circulation 1999; 100: 999-1008.

10. Zannad F, McMurray JJ, Krum H, et al. EMPHASIS-HF Study Group. Eplerenone in patients with systolic heart failure and mild symptoms. N Engl J Med 2011; 364(1): 11-21.

11. Jankowska EA, Biel B, Majda J, et al. Anabolic deficiency in men with chronic heart failure: prevalence and detrimental impact on survival. Circulation 2006; 114: 1829-37.

12. Saccà L. Heart failure as a multiple hormonal deficiency syndrome. Circ Heart Fail 2009; 2: 151-156.

13. Cittadini A, Saldamarco L, Marra AM, et al. Growth hormone deficiency in patients with chronic heart failure and beneficial effects of its correction. J Clin Endocrinol Metab 2009; 94(9): 3329-36.

14. Cittadini A, Bossone E, Marra AM, Arcopinto M, Bobbio $\mathrm{E}$ et al. Anabolic/catabolic imbalance in chronic heart failure. Monaldi Arch Chest Dis 2010; 74(2): 53-6.

15. Marra AM, Arcopinto M, Bossone E et al. Cardiovascular abnormalities in the Low IGF-1 Syndrome: Insight from the T.O.S.CA. Project. Eur Heart J 2010; 31: 28081 (Abstract Supplement).

16. Williams SC, Schmaltz SP, Morton DJ, Koss RG, Loeb JM. Quality of care in U.S. hospitals as reflected by standardized measures, 2002-2004. N Engl J Med 2005; 353: 255-264.

17. Lee DS, Tu JV, Juurlink DN, et al. Risk-treatment mismatch in the pharmacotherapy of heart failure. JAMA 2005; 294: 1240-1247.

18. Fonarow GC, Yancy CW, Heywood JT, et al for the ADHERE Scientific Advisory Committee, Study Group, and Investigators. Adherence to heart failure quality-of-care indicators in US hospitals: analysis of the ADHERE Registry. Arch Intern Med 2005; 165: 1469-1477.

19. Shah B, Hernandez AF, Liang L, et al. For the Get With the Guidelines Steering Committee and Hospitals. Variation and characteristics of implantable cardioverter defibrillator use in patients with heart failure: data from the GWTG-HF (Get With The Guidelines-Heart Failure) registry. J Am Coll Cardiol. 2009; 53: 416-422.

20. Piccini JP, Hernandez AF, Dai D, et al. For the Get With the Guidelines Steering Committee and Hospitals. Use of cardiac resynchronization therapy in patients hospitalized with heart failure. Circulation 2008; 118: 926-933.

21. Stafford RS, Radley DC. The underutilization of cardiac medications of proven benefit, 1990 to 2002. J Am Coll Cardiol 2003; 41: 56-61.

22. Fonarow GC, Yancy CW, Albert NM, et al. Heart failure care in the outpatient cardiology practice setting: findings from IMPROVE HF. Circ Heart Fail 2008; 1: 98-106.

23. American College of Cardiology: Heart Failure Gap project in Oregon. Available at: http://www.cardiologyinoregon.org/information/information.html. Accessed 22 January 2011.

24. American Heart Association: Get With the Guidelines-Heart Failure. Available at: http://www.heart.org/HEARTORG/ HealthcareResearch/GetWithTheGuidelinesHFStroke/Get WithTheGuidelinesHeartFailureHomePage/Get-With-TheGuidelines-Heart-Failure-Overview_UCM_307806_Article.jsp. Accessed 22 January 2011.
25. Sutherland K (2010) Bridging the quality gap: Heart failure. The Health Foundation. Available at: http://www.health. org.uk/publications/bridging-the-quality-gap-heart-failure/. Accessed 22 January 2011.

26. Fonarow GC, Abraham WT, Albert NM, et al. For the OPTIMIZE-HF Investigators and Hospitals. Influence of a performance-improvement initiative on quality of care for patients hospitalized with heart failure: results of the Organized Program to Initiate Lifesaving Treatment in Hospitalized Patients With Heart Failure (OPTIMIZE-HF). Arch Intern Med 2007; 167: 1493-1502.

27. Fonarow GC, Heywood JT, Heidenreich PA, Lopatin M, Yancy CW. For the ADHERE Scientific Advisory Committee and Investigators. Temporal trends in clinical characteristics, treatments, and outcomes for heart failure hospitalizations, 2002 to 2004: findings from Acute Decompensated Heart Failure National Registry (ADHERE). Am Heart J 2007; 153: 1021-1028.

28. Gregg C, Fonarow GC, Nancy M, et al. Improving Evidence-Based Care for Heart Failure in Outpatient Cardiology Practices Primary Results of the Registry to Improve the Use of Evidence-Based Heart Failure Therapies in the Outpatient Setting (IMPROVE HF). Circulation 2010; 122: 585-596.

29. Agabiti N, Pirani M, Schifano P et al. Italian Study Group on Inequalities in Health Care. Income level and chronic ambulatory care sensitive conditions in adults: a multicity population-based study in Italy. BMC Public Health 2009; 11; 9: 457.

30. Stewart S, Jenkins A, Buchan S, McGuire A, Capewell S, McMurray JJ. The current cost of heart failure to the National Health Service in the UK. Eur J Heart Fail 2002; 4: 361-371.

31. Stewart S. Financial aspects of heart failure programs of care. Eur J Heart Fail 2005; 7: 423-428.

32. Bellotti P, Badano LP, Acquarone N et al. Specialty-related differences in the epidemiology, clinical profile, management and outcome of patients hospitalised for heart failure: the OSCUR study. Eur Heart J 2001; 22(7): 596-604.

33. Consensus Conference: Il percorso assistenziale del paziente con scompenso cardiaco. G Ital Cardiol 2006; 7(6): 387-432.

34. Cleveland Clinic Heart failure management program available at: http://my.clevelandclinic.org/heart/disorders/heartfailure/hfmanagement.aspx Accessed 22 January 2011.

35. Bollettino ufficiale della regione Campania n. 8 del 9 (Febbraio 2009) Modifiche e integrazioni alla deliberazione $n^{\circ} 2040$ del 23 dicembre 2008 "Modello organizzativo del Day Service" (DGRC 546/2007). Available at: http://www.sito.regione.campania.it/burc/pdf09/burc08or _09/del102_09.pdf Accessed 22 January 2011.

36. Bollettino ufficiale della regione Campania n. 546 del 23 (Aprile 2007) Allegato 2: regolamentazione dei ricoveri in Day Hospital di tipo medico. Available at: http://www.sito.regione.campania.it/burc/pdf07/burc23or _07/del546_07.pdf Accessed 22 January 2011.

37. Quaderni del Ministero della Salute $n^{\circ} 1$ gennaio-febbraio (2010). Criteri di appropriatezza clinica, tecnologica e strutturale nell'assistenza alle malattie del sistema cardiovascolare. La Rete per il cardiopatico cronico: organizzazione della Rete del paziente cronico pag. 33-38. Available at: http://www.quadernidellasalute.it/archivio-quaderni/ 1-gennaio-febbraio-2010. Accessed 22 January 2011.

38. Charron P, Arad M, Arbustini E, et al. Genetic counselling and testing in cardiomyopathies: a position statement of the European Society of Cardiology Working Group on Myocardial and Pericardial Diseases. Eur Heart $J$ 2010; 31(22): 2715-26.

39. Hershberger RE, Lindenfeld J, Mestroni L, Seidman CE, Taylor MR, Towbin JA. Heart Failure Society of America. Genetic evaluation of cardiomyopathy-a Heart Failure Society of America practice guideline. J Card Fail 2009; 15(2): 83-97. 


\section{The T.O.S.CA. Investigators}

Eduardo Bossone, Rodolfo Citro, Vincenzo De Paola, Giuseppe Di Benedetto, Alberto Gigantino, Francesco Silvestri, Azienda Ospedaliera-Universitaria San Giovanni di Dio e Ruggi d'Aragona, Salerno, Italy; Luigi Caliendo, San Leonardo Hospital, Castellammare di Stabia, Italy; Vincenzo Cirrincione, Graziella Malizia, Azienda Ospedaliera Villa Sofia-Cervello, Palermo, Italy; Alessandro Frigiola, Andrea Ballotta, Carlo De Vincentis, Tommaso Generali, Francesca Giacomazzi, I.R.C.C.S. Policlinico San Donato, San Donato, Italy; Jorgen Isgaard, Odd Bech-Hanssen, Entela Bollano, Kristjan Karason, Sahlgrenska University Hospital, Göteborg, Sweden; Giuseppe Limongelli, Paolo Calabrò, Raffaele Calabrò, Antonello D’Andrea, Valeria Maddaloni, Giuseppe Pacileo, Raffaella Scarafile, Seconda Università degli Studi di Napoli, Napoli, Italy; Salvatore Milano, Gabriella Misiano, Policlinico P. Giaccone, Palermo, Italy; Mario Polverino, Valentina Musella, Carlo Santoriello, Azienda Sanitaria Locale di Salerno; Saverio Rega, D’Ambrosio Giuseppe, Martiri del Villa Marta, Sarno, Italy; Luigi Saccà, Antonio Cittadini, Michele Arcopinto, Emanuele Bobbio, Domenico Sirico, Francesco Ferrara, Alberto Maria Marra, Maria Gaia Monti, Lavinia Saldamarco, Andrea Salzano, Dario Celentani, Daniele Luiso, Emauele Spina, Andrea Giacomo Campanino, Carmen Miele, Luca Formicola, Maria Laura Santopaolo, Davide Della Polla, Università Federico II di Napoli, Napoli, Italy; Olga Vriz, Francesco Graniero, Giancarla Marcuzzi, Gemma Schiaulini, San Antonio Hospital, San Daniele del Friuli, Italy.

Data Management and Biostatistical Support

Armando Mazzeo, Annapaola Cirillo, Cooperativa "Cippus”, Nola, Italy. 